Santé mentale au Québec

Améliorer la détection et le traitement des psychoses débutantes au Québec : L’Association québécoise des programmes pour premiers épisodes psychotiques (AQPPEP) y voit

Improving detection and treatment of early psychosis in Québec: the Quebec association of early psychosis (l'Association québécoise des programmes pour premiers épisodes psychotiques, AQPPEP), sees to it

Mejorar la detección y el tratamiento de las psicosis iniciales en Quebec: La Asociación Quebequense de Programas Para los Primeros Episodios Psicóticos (AQPPEP) se encarga de ello

Melhorar a detecção e o tratamento de psicoses inicial no Quebec: criação da Associação Quebequense dos Programas para os Primeiros Episódios Psicóticos (AQPPEP)

Sophie L'Heureux, Luc Nicole, Amal Abdel-Baki, Marc-André Roy, Nathalie Gingras et Marie-France Demers

Volume 32, numéro 1, printemps 2007

La psychothérapie focalisée sur le transfert : Québec-New York (1) et Windigo I (2)

URI : https://id.erudit.org/iderudit/016522ar

DOI : https://doi.org/10.7202/016522ar

Aller au sommaire du numéro

Éditeur(s)

Revue Santé mentale au Québec

ISSN

0383-6320 (imprimé)

1708-3923 (numérique)

Découvrir la revue

Citer ce document

L'Heureux, S., Nicole, L., Abdel-Baki, A., Roy, M.-A., Gingras, N. \& Demers, M.-F. (2007). Améliorer la détection et le traitement des psychoses débutantes au Québec : L’Association québécoise des programmes pour premiers épisodes psychotiques (AQPPEP) y voit. Santé mentale au Québec, 32(1), 299-315. https://doi.org/10.7202/016522ar

\section{Résumé de l'article}

Juin 2004 : l'AQPPEP (Association québécoise des programmes pour premiers épisodes psychotiques) (http://www.aqppep.com/) voit le jour. Son rôle est de favoriser les échanges cliniques et scientifiques entre les professionnels et les chercheurs qui s'intéressent aux personnes atteintes de psychose débutante, et de permettre une détection plus précoce de la psychose. Elle vise également une prise de conscience de cette problématique par la population et les instances gouvernementales. À cette fin, l'AQPPEP a organisé la première journée québécoise de sensibilisation à la psychose, et développé un des rares sites internet francophones dans ce domaine. Dans un esprit de concertation, l'Association constitue un outil pour affronter les problèmes que plusieurs cliniques pour premiers épisodes psychotiques rencontrent, pour partager et développer des solutions communes entre elles. 


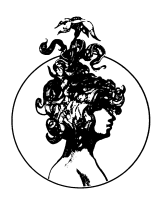

\title{
Améliorer la détection et le traitement des psychoses débutantes au Québec: L'Association québécoise des programmes pour premiers épisodes psychotiques (AQPPEP) y voit
}

\author{
Sophie L'Heureux* \\ Luc Nicole** \\ Amal Abdel-Baki ${ }^{* * *}$ \\ Marc-André Roy ${ }^{\star * * *}$ \\ Nathalie Gingras ${ }^{\star * * *}$ \\ Marie-France Demers ${ }^{\star * \star \star \star *}$
}

Juin 2004: 1'AQPPEP (Association québécoise des programmes pour premiers épisodes psychotiques) (http://www.aqppep.com/) voit le jour. Son rôle est de favoriser les échanges cliniques et scientifiques entre les professionnels et les chercheurs qui s'intéressent aux personnes atteintes de psychose débutante, et de permettre une détection plus précoce de la psychose. Elle vise également une prise de conscience de cette problématique par la population et les instances gouvernementales. À cette fin, l'AQPPEP a organisé la première journée québécoise de sensibilisation à la psychose, et développé un des rares sites internet francophones dans ce domaine. Dans un esprit de concertation, l'Association constitue un outil pour affronter les problèmes que plusieurs cliniques pour premiers épisodes psychotiques rencontrent, pour partager et développer des solutions communes entre elles.

partir des années 80 , les équipes australiennes et britanniques ont
identifié que les jeunes patients qui présentent une psychose
débutante ont des besoins spécifiques nécessitant une prise en charge
distincte de celle des personnes atteintes de schizophrénie, dont la
maladie présente plusieurs années d'évolution (McGorry, 2000;

\footnotetext{
* MD, FRCPC, Clinique Notre-Dame des Victoires, CHRG, Québec

** MD FRCPC, M. Sc., Clinique des jeunes adultes, HLHL, Montréal

*** MD FRCPC, M. Sc., Clinique JAP, CHUM, Montréal

**** MD, FRCPC, M. Sc., Clinique Notre-Dame des Victoires, CHRG, Québec

***** MD FRCPC, M. Sc., Clinique DIPP, Centre de pédopsychiatrie du CHUQ, Québec

****** B Pharm MSc, Clinique Notre-Dame des Victoires, CHRG, Québec
} 
Birchwood, 2000). L'emphase fut mise sur la détection précoce des symptômes de psychose (Falloon, 1992) et l'offre la plus rapide possible d'une intervention spécialisée et intensive, afin de permettre un contrôle efficace des symptômes et de limiter les conséquences négatives de la psychose. L'un des principaux objectifs de l'intervention précoce est la réduction de la durée de psychose non traitée (DPNT), soit le délai entre le début des symptômes psychotiques et l'instauration d'un traitement adéquat (Larsen, 2001). Ce délai, qui se chiffre en moyenne à un an (Birchwood, 2000), est associé à un mauvais pronostic symptomatique et fonctionnel (Linszen et al., 1998; McGlashan, 1999; Harrigan, 2003). Dans ce contexte, des efforts croissants au niveau international ont été consacrés à l'instauration de programmes spécifiques pour premiers épisodes psychotiques, certains guides de pratique étant même développés dans cet objectif (Edwards et al., 2002; Ehmann et al., 2004). L'intervention précoce dans le cadre de ces programmes vise, autant que possible, à prévenir la détérioration psychosociale habituellement observée durant les premières années d'évolution de la maladie, voire même avant le premier épisode psychotique. Une approche de traitement intensif dès les premières difficultés de fonctionnement social, familial et professionnel permet au mieux d'éviter cette détérioration, sinon d'en amoindrir les effets et d'optimiser l'accomplissement d'un projet de vie (Abdel-Baki, 2006, Malla et al., 2001). Elle stimule l'espoir chez le jeune et sa famille devant une maladie qui peut être très dévastatrice, et qui peut représenter en soi un «traumatisme» (McGorry et al, 1991). En ce sens, couplée à l'intervention familiale, c'est la thérapie d'orientation cognitivo-comportementale qui s'est révélée la plus indiquée. Elle a été adoptée à travers le monde par presque toutes les cliniques pour premiers épisodes psychotiques (Addington et al., 2005).

Au Québec, la Clinique des Jeunes Adultes a vu le jour à Montréal en 1987 grâce à l'initiative d'un groupe de professionnels en psychiatrie motivés. Durant les années qui ont suivi, d'autres programmes ont été mis sur pied, d'abord dans les régions de Montréal et de Québec, puis ce mouvement s'est étendu à toute la province. Ces cliniques peuvent s'adresser tant à une population adulte qu'adolescente, vu l'âge du début de la maladie. Les programmes se sont développés indépendamment les uns des autres, tributaires uniquement de la motivation des équipes en place et des moyens autorisés par les organisations hospitalières dont ils relevaient. Considérant les besoins de la population et la nécessité d'une meilleure communication entre les personnes oeuvrant vers le même objectif, il est devenu impératif de créer un réseau d'échanges entre les différentes cliniques. L'IEPA (International Early Psychosis Association) avait vu le jour en 1998, regroupant des intervenants intéressés par 
cette problématique, avec un congrès international aux deux ans. Dans cette lignée mais au niveau provincial, l'AQPPEP (Association québécoise des programmes pour premiers épisodes psychotiques) a été fondée en juin 2004 par des membres de cinq cliniques pour psychose débutante de Montréal et de Québec (http://www.aqppep.com/). L'association regroupe maintenant des membres provenant non seulement des grands centres, mais aussi des régions où de telles initiatives se développent.

\section{Objectifs de l'Association}

L'Association a pour but d'optimiser la collaboration et le partage d'expertise entre les différentes cliniques, d'aider au développement de nouvelles cliniques dans la province, de travailler conjointement à permettre une détection plus précoce de la psychose, de même que d'améliorer la prise de conscience de la problématique par la population et par les instances gouvernementales. Des objectifs cliniques, d'enseignement et de recherche ont été intégrés à la mission de l'Association (voir tableau 1).

\section{Membres}

Durant sa première année d'existence, l'AQPPEP a cherché à se faire connaître afin de recruter des membres potentiels, soit tous les professionnels œuvrant à l'intérieur de cliniques pour premiers épisodes psychotiques (membres actifs) ainsi que tout professionnel ayant un intérêt particulier pour le sujet (membres associés). À cet effet, des intervenants de toutes les cliniques connues ont été contactés, un feuillet d'information a été distribué avec le journal de l'Association des médecins psychiatres du Québec, ainsi que mention de l'Association à diverses conférences et réunions (L'Heureux et al., 2006). Ainsi, l'AQPPEP vise à rejoindre des intervenants dans toutes les régions de la province, et à regrouper des intervenants de formations professionnelles diversifiées (voir tableau 2).

Les cliniques pour premiers épisodes psychotiques membres de l'AQPPEP ont une mission en lien avec celle de l'association. Elles partagent une vision commune sur les principes de base d'accessibilité rapide aux soins, et de spécificité de l'approche pour la période qui entoure le premier épisode de psychose. Elles ont cependant chacune leur indépendance, d'où une certaine variabilité dans leurs critères d'inclusion et dans leur fonctionnement, tel qu'il est retrouvé au niveau international (Edwards et al., 2002; Ehmann et al., 2004) (tableau 3). Une évaluation multidisciplinaire initiale est faite pour s'assurer de 


\section{Tableau 1}

\section{Objectifs professionnels et moraux de l'AQPPEP (tirés des statuts de règlements de l'AQPPEP) ${ }^{1}$}

\begin{tabular}{|l|l|}
\hline $\begin{array}{l}\text { Objectifs } \\
\text { professionnels }\end{array}$ & $\begin{array}{l}\text { Organiser des activités d'échanges scienti- } \\
\text { fiques en rapport avec les besoins de ses } \\
\text { membres. } \\
\text { Favoriser l'amélioration des services au niveau } \\
\text { de la détection précoce ainsi que de l'accessi- } \\
\text { bilité et la qualité des soins. } \\
\text { Optimiser les interventions offertes aux per- } \\
\text { sonnes atteintes de premier épisode psycho- } \\
\text { tique et à leurs proches. } \\
\text { Favoriser le développement de nouveaux pro- } \\
\text { grammes cliniques destinés aux personnes } \\
\text { atteintes de premier épisode psychotique ainsi } \\
\text { que le développement de programmes de } \\
\text { détection précoce et de suivi de personnes à } \\
\text { haut risque de développer une psychose, tant } \\
\text { au niveau de la clientèle infantile, juvénile } \\
\text { qu'adulte. } \\
\text { Promouvoir le développement de la recherche } \\
\text { et sa diffusion auprès des professionnels et des } \\
\text { étudiants sur les problématiques reliées aux } \\
\text { personnes présentant leurs premiers épisodes } \\
\text { psychotiques. } \\
\text { Participer à la sensibilisation du grand public } \\
\text { en vue de diminuer les stigmates reliés au pre- } \\
\text { mier épisode psychotique et d'améliorer } \\
\text { l'identification et la référence des personnes } \\
\text { atteintes de premier épisode psychotique à des } \\
\text { services appropriés. } \\
\text { Maximiser les collaborations avec les diffé- } \\
\text { rents partenaires du réseau, particulièrement } \\
\text { les Associations de proches et de personnes } \\
\text { atteintes. } \\
\text { Sensibiliser les administrateurs du réseau de la } \\
\text { santé, les autorités politiques ainsi que la } \\
\text { population à l'importance des enjeux reliés au } \\
\text { PEP en vue d'obtenir les ressources néces- } \\
\text { saires à l'atteinte des objectifs ci-haut men- } \\
\text { tionnés. }\end{array}$ \\
\hline
\end{tabular}




\begin{tabular}{|l|l|}
\hline Objectif moral & $\begin{array}{l}\text { Veiller à ce que la pratique de la psychiatrie } \\
\text { dans les programmes traitant les personnes } \\
\text { atteintes de premiers épisodes psychotiques } \\
\text { respecte la déontologie en vigueur et l'éthique. }\end{array}$ \\
\hline
\end{tabular}

Tableau 2

Description des membres de l'AQPPEP

\begin{tabular}{|c|c|}
\hline $\begin{array}{l}\text { Nombre de membres de } \\
\text { l'AQPPEP } \\
\text { (2005-2006) }\end{array}$ & $\begin{array}{l}76 \text { membres: } \\
59 \text { membres actifs } \\
17 \text { membres associés }\end{array}$ \\
\hline $\begin{array}{l}\text { Description des membres } \\
\text { actifs }\end{array}$ & $\begin{array}{l}59 \text { membres actifs provenant de } 11 \\
\text { cliniques: } \\
7 \text { cliniques affiliées à des centres } \\
\text { hospitaliers universitaires } \\
4 \text { cliniques d'hôpitaux régionaux }\end{array}$ \\
\hline $\begin{array}{l}\text { Description des membres } \\
\text { associés }\end{array}$ & $\begin{array}{l}17 \text { membres associés provenant de } \\
12 \text { affiliations différentes: } \\
7 \text { hôpitaux régionaux } \\
3 \text { organismes de santé mentale } \\
2 \text { écoles }\end{array}$ \\
\hline Type de professionnels & $\begin{array}{l}30 \text { psychiatres } \\
11 \text { ergothérapeutes } \\
10 \text { psychologues } \\
8 \text { travailleurs sociaux } \\
7 \text { infirmières } \\
4 \text { éducateurs } \\
2 \text { pharmaciens } \\
2 \text { intervenants scolaires } \\
\text { (directeur/conseiller pédagogique) } \\
2 \text { assistant de recherche/gestionnaire }\end{array}$ \\
\hline Type de population desservie & $\begin{array}{l}68 \text { membres travaillant auprès de la } \\
\text { population adulte } \\
8 \text { membres travaillant auprès de la } \\
\text { population pédopsychiatrique (enfants } \\
\text { et adolescents) }\end{array}$ \\
\hline
\end{tabular}

l'indication des interventions, et préciser les besoins spécifiques de la personne. L'évaluation touche plusieurs niveaux: diagnostic, bilan métabolique et organique, profil pharmacologique, fonctionnement social et occupationnel, capacités cognitives, aspects familiaux. Les 
Tableau 3

Description des cliniques pour premiers épisodes psychotiques québécoises

\begin{tabular}{|c|c|c|c|c|c|c|}
\hline Clinique & Ville & Population & $\begin{array}{c}\text { Critères } \\
\text { d'inclusion }\end{array}$ & $\begin{array}{c}\text { Critères } \\
\text { d'exclusion }\end{array}$ & Enseignement & Recherche \\
\hline \begin{tabular}{|l|} 
Clinique des \\
jeunes adultes \\
(Hôpital LHL)
\end{tabular} & Montréal & 400000 & $\begin{array}{l}-18-30 \text { ans } \\
\text {-Schizophrénie } \\
\text { ou psychose } \\
\text { apparentée } \\
\text { - Non traitée ou } \\
\text { traitée depuis } \\
\text { moins de } 1 \text { an }\end{array}$ & $\begin{array}{l}\text { Déficience } \\
\text { intellectuelle } \\
\text { modérée ou } \\
\text { sévère }\end{array}$ & $\begin{array}{l}\text { Supervision des } \\
\text { résidents en psychiatrie, } \\
\text { externes en médecine et } \\
\text { stagiaires (service } \\
\text { social, ergothérapie, } \\
\text { sciences infirmières, } \\
\text { orientation-counseling, } \\
\text { éducation spécialisée) }\end{array}$ & $\begin{array}{l}\text {-Évolution: ESPÉUM- } \\
\text { Étude de Suivi } \\
\text { Psychose Émergente } \\
\text { de l'Université de } \\
\text { Montréal (en } \\
\text { collaboration avec } \\
\text { l'équipe de la clinique } \\
\text { JAP du CHUM) } \\
\text { - Pharmacothérapie } \\
\text { - Interventions } \\
\text { psychosociales } \\
\text { (individuelles, de } \\
\text { groupe, familiales) } \\
\text { - Génétique } \\
\text { - Comorbidité } \\
\text { - Neuropsychologie } \\
\text { - Organisation des } \\
\text { services }\end{array}$ \\
\hline
\end{tabular}




\begin{tabular}{|c|c|c|c|c|c|c|}
\hline Clinique & Ville & Population & $\begin{array}{c}\text { Critères } \\
\text { d'inclusion }\end{array}$ & $\begin{array}{c}\text { Critères } \\
\text { d'exclusion }\end{array}$ & Enseignement & Recherche \\
\hline $\begin{array}{l}\text { Clinique JAP } \\
\text { (Hôpital Saint- } \\
\text { Luc, CHUM) }\end{array}$ & Montréal & 200000 & $\begin{array}{l}-18-30 \text { ans } \\
\text { - Psychose } \\
\text { affective ou non } \\
\text { affective } \\
\text { - Non traitée ou } \\
\text { traitée depuis } \\
\text { moins de } 1 \text { an }\end{array}$ & $\begin{array}{l}\text { Déficience } \\
\text { intellectuelle }\end{array}$ & $\begin{array}{l}\text { Supervision des } \\
\text { résidents en psychiatrie, } \\
\text { externes en médecine et } \\
\text { stagiaires (service } \\
\text { social, ergothérapie, } \\
\text { sciences infirmières) }\end{array}$ & $\begin{array}{l}\text {-Évolution : ESPEUM } \\
\text { - Thérapies } \\
\text { psychosociales } \\
\text { - Insertion au travail } \\
\text { - Organisation des } \\
\text { services }\end{array}$ \\
\hline $\begin{array}{l}\text { Clinique du } \\
\text { Pavillon blanc } \\
\text { (Hôpital du Sacré- } \\
\text { Cœur) }\end{array}$ & Montréal & 325000 & $\begin{array}{l}-18-30 \text { ans } \\
- \text { Moins de } 5 \text { ans } \\
\text { d'évolution }\end{array}$ & $\begin{array}{l}\text { Déficience } \\
\text { intellectuelle }\end{array}$ & $\begin{array}{l}\text { Supervision des } \\
\text { externes en médecine et } \\
\text { stagiaires (service } \\
\text { social, ergothérapie) }\end{array}$ & $\begin{array}{l}\text { - Prodrome } \\
\text { - Insight } \\
\text { - Insertion au travail }\end{array}$ \\
\hline $\begin{array}{l}\text { Clinique } \\
\text { d'évaluation et } \\
\text { d'intervention du } \\
\text { premier épisode } \\
\text { psychotique } \\
\text { (Centre des } \\
\text { adolescents du } \\
\text { PAP, Hôpital du } \\
\text { Sacré-Coeur) }\end{array}$ & Montréal & $\begin{array}{l}120000 \\
(0-18 \text { ans })\end{array}$ & $\begin{array}{l}-13-18 \text { ans } \\
- \text { Psychose } \\
\text { affective ou non } \\
\text { affective, } \\
\text { toxique }\end{array}$ & $\begin{array}{l}\text { Déficience } \\
\text { intellectuelle }\end{array}$ & $\begin{array}{l}\text { Supervision des } \\
\text { résidents en psychiatrie, } \\
\text { externes en médecine et } \\
\text { stagiaires (psychologie, } \\
\text { service social) }\end{array}$ & $\begin{array}{l}\text {-Neuropsychologie } \\
\text {-Évolution }\end{array}$ \\
\hline
\end{tabular}




\begin{tabular}{|c|c|c|c|c|c|c|}
\hline Clinique & Ville & Population & $\begin{array}{c}\text { Critères } \\
\text { d'inclusion }\end{array}$ & $\begin{array}{c}\text { Critères } \\
\text { d'exclusion }\end{array}$ & Enseignement & Recherche \\
\hline $\begin{array}{l}\text { Programme PEPP } \\
\text { (Hôpital Douglas) }\end{array}$ & Montréal & 290000 & $\begin{array}{l}-14-30 \text { ans } \\
\text { - Psychose } \\
\text { affective ou non } \\
\text { affective } \\
\text { - Non traitée ou } \\
\text { traitée depuis } \\
\text { moins de } 30 \\
\text { jours }\end{array}$ & $\begin{array}{l}\text { - Trouble } \\
\text { envahissant du } \\
\text { développement } \\
\text { - Lésion cérébrale } \\
\text { - Déficience } \\
\text { intellectuelle } \\
\text {-Épilepsie } \\
\text { - Antécédents } \\
\text { sévères légaux }\end{array}$ & $\begin{array}{l}\text { Supervision des } \\
\text { résidents/fellows en } \\
\text { psychiatrie et stagiaires } \\
\text { (ergothérapie, sciences } \\
\text { infirmières, } \\
\text { psychologie) } \\
\text { Enseignement } \\
\text { universitaire dans les } \\
\text { mêmes disciplines } \\
\text { - Facteurs de risque } \\
\text { pour la psychose } \\
\text { - Prédicteurs d'évolution } \\
\text { à long terme }\end{array}$ & $\begin{array}{l}\text { - Imagerie cérébrale, } \\
\text { symptômes négatif et } \\
\text { insight } \\
\text { - Génétique } \\
\text { - Interventions } \\
\text { psychosociales } \\
\text { - Pharmacothérapie } \\
\text { - Qualité de la vie }\end{array}$ \\
\hline $\begin{array}{l}\text { Clinique Notre- } \\
\text { Dame des } \\
\text { Victoires (CHRG) }\end{array}$ & Québec & 500000 & $\begin{array}{l}-18-35 \text { ans } \\
\text { - Psychose non } \\
\text { affective } \\
\text { - Non traitée ou } \\
\text { traitée depuis } \\
\text { moins de } 1 \text { an }\end{array}$ & $\begin{array}{l}\text { - Déficience } \\
\text { intellectuelle } \\
\text { modérée ou } \\
\text { sévère } \\
\text { - Psychose } \\
\text { exclusivement } \\
\text { toxique }\end{array}$ & $\begin{array}{l}\text { - Supervision des } \\
\text { résidents en } \\
\text { psychiatrie et } \\
\text { stagiaires (service } \\
\text { social, ergothérapie, } \\
\text { sciences infirmières, } \\
\text { psychologie, } \\
\text { pharmacie) } \\
\text { - Enseignement } \\
\text { universitaire dans les } \\
\text { mêmes disciplines } \\
\text { - Pharmacologie } \\
\text { - Interventions } \\
\text { psychosociales }\end{array}$ & $\begin{array}{l}\text { (individuelles, de } \\
\text { groupe, familiales) } \\
\text {-Comorbidité anxieuse } \\
\text { - Génétique } \\
\text { - Neuropsychologie }\end{array}$ \\
\hline
\end{tabular}




\begin{tabular}{|c|c|c|c|c|c|c|}
\hline Clinique & Ville & Population & $\begin{array}{c}\text { Critères } \\
\text { d'inclusion }\end{array}$ & $\begin{array}{c}\text { Critères } \\
\text { d'exclusion }\end{array}$ & Enseignement & Recherche \\
\hline $\begin{array}{l}\text { Clinique DIPP } \\
\text { (CHUQ) }\end{array}$ & Québec & 500000 & $\begin{array}{l}-\leq 18 \text { ans } \\
- \text { Psychose } \\
\text { affective ou non } \\
\text { affective, } \\
\text { toxique } \\
\text { - Prodrome }\end{array}$ & & $\begin{array}{l}\text { - Supervision des } \\
\text { résidents en } \\
\text { psychiatrie et } \\
\text { stagiaires (service } \\
\text { social, ergothérapie, } \\
\text { psychologie) }\end{array}$ & $\begin{array}{l}\text { - Thérapie cognitivo- } \\
\text { comportementale des } \\
\text { psychoses } \\
\text { - Neuropsychologie } \\
\text { - Prédicteurs } \\
\text { d'évolution } \\
\text { - Caractéristiques } \\
\text { cliniques des épisodes } \\
\text { chez l'enfant et } \\
\text { l'adolescent }\end{array}$ \\
\hline Programme RAP & Rimouski & 110000 & $\begin{array}{l}-18-40 \text { ans- } \\
- \text { Tout type de } \\
\text { psychose }\end{array}$ & $\begin{array}{l}\text { Déficience } \\
\text { intellectuelle }\end{array}$ & $\begin{array}{l}\text { Supervision des } \\
\text { résidents en psychiatrie, } \\
\text { en médecine familiale } \\
\text { et des externes en } \\
\text { médecine }\end{array}$ & $-\grave{A}$ venir \\
\hline $\begin{array}{l}\text { Clinique } \\
\text { spécialisée jeunes } \\
\text { adultes }\end{array}$ & $\begin{array}{l}\text { Drummondv } \\
\text { ille }\end{array}$ & 90000 & $\begin{array}{l}-16-30 \text { ans } \\
- \text { Psychose non } \\
\text { affective }\end{array}$ & $\begin{array}{l}\text { - Déficience } \\
\text { intellectuelle } \\
\text { - Psychose } \\
\text { toxique }\end{array}$ & Non & Non \\
\hline $\begin{array}{l}\text { Programme des } \\
\text { premières } \\
\text { psychoses }\end{array}$ & Saguenay & 150000 & $\begin{array}{l}-18-30 \text { ans } \\
- \text { Psychose } \\
\text { affective ou non } \\
\text { affective } \\
\text { - Moins de } 5 \text { ans } \\
\text { d'évolution }\end{array}$ & $\begin{array}{l}\text { Déficience } \\
\text { intellectuelle } \\
\text { modérée ou } \\
\text { sévère }\end{array}$ & $\begin{array}{l}\text { Supervision des } \\
\text { externes en médecine et } \\
\text { stagiaires (sciences } \\
\text { infirmières, } \\
\text { ergothérapie) }\end{array}$ & Non \\
\hline
\end{tabular}




\begin{tabular}{|c|c|c|c|c|c|c|}
\hline Clinique & Ville & Population & $\begin{array}{c}\text { Critères } \\
\text { d'inclusion }\end{array}$ & $\begin{array}{c}\text { Critères } \\
\text { d'exclusion }\end{array}$ & Enseignement & Recherche \\
\hline $\begin{array}{l}\text { Programme pour } \\
\text { jeunes } \\
\text { psychotiques }\end{array}$ & Shawinigan & 75000 & $\begin{array}{l}-16-30 \text { ans } \\
\text { - Psychose } \\
\text { affective ou non } \\
\text { affective } \\
\text { - Sans traitement } \\
\text { de réadaptation } \\
\text { antérieur }\end{array}$ & $\begin{array}{l}\text { - Trouble sévère } \\
\text { de la } \\
\text { personnalité au } \\
\text { premier plan } \\
\text { - Déficience } \\
\text { intellectuelle } \\
\text { modérée ou } \\
\text { sévère. } \\
\text { - Maladie } \\
\text { organique } \\
\text { primaire } \\
\text { - Intoxication } \\
\text { aiguë aux } \\
\text { substances }\end{array}$ & $\begin{array}{l}\text { - Supervision des } \\
\text { externes en médecine } \\
\text { et stagiaires } \\
\text { (ergothérapie, } \\
\text { psychologie) }\end{array}$ & $\begin{array}{l}\text { - Liens d'attachement } \\
\text { - Lien entre fonctions } \\
\text { cognitives et } \\
\text { fonctionnement social } \\
\text { et professionnel }\end{array}$ \\
\hline
\end{tabular}


interventions thérapeutiques sont ensuite déterminées en fonction de ces éléments. Elles incluent la pharmacothérapie, le suivi individuel et de groupe d'orientation cognitive avec psychoéducation, la thérapie motivationnelle et divers groupes thérapeutiques et de soutien (ergothérapie, habiletés sociales, résolution de problèmes, gestion de l'anxiété, etc.), l'intervention familiale, la réadaptation vocationnelle (souvent en lien avec une ressource spécialisée externe). Certaines cliniques intègrent des volets hôpital de jour, «case management » ou intervention intensive dans la communauté.

\section{Réalisations de l'AQPPEP 2004-2006}

Les premières réalisations de l'AQPPEP ont été : 1) L'organisation d'une première journée de sensibilisation à la psychose, à Montréal, qui visait les personnes travaillant auprès des jeunes et la population générale ; 2) Le développement de l'un des premiers sites web francophones sur le sujet des psychoses débutantes.

\section{Journée de sensibilisation à la psychose}

La première journée de sensibilisation à la psychose débutante s'est tenue à Montréal le 11 novembre 2005. L'organisation de cette journée était en ligne directe avec la mission de l'Association, qui vise à promouvoir une meilleure détection de la psychose dans la population générale, et à sensibiliser les instances gouvernementales à cette problématique. Des invitations ont été envoyées à diverses organisations œuvrant auprès de jeunes pouvant présenter un premier épisode psychotique, comme des écoles, des travailleurs sociaux et des psychologues. M. Philippe Couillard, ministre de la Santé au Québec, a inauguré la journée par une allocution qui soulignait l'importance de ce travail. Différentes conférences ont été données au cours de la journée sur les symptômes de psychose et son prodrome (état mental à risque), les difficultés liées à la détection précoce, le rôle crucial de l'intervention précoce pour le pronostic, la comorbidité fréquente avec la toxicomanie, et les processus de réadaptation. Entre les conférences, plusieurs kiosques ont été présentés et ont traité d'un domaine spécifique (médication, approche aux familles, etc.). Ces kiosques étaient animés par des membres des cliniques et organismes communautaires; ils ont permis des échanges plus personnels avec les participants.

Quatre cent trente-sept (437) personnes ont participé à cette première journée de sensibilisation, dont soixante-trois (63) bénévoles agissant comme conférenciers, animateurs de kiosque ou impliqués dans l'organisation de la journée. 
Tout en étant satisfaits de ce niveau de participation, l'Association est consciente qu'une sensibilisation du public est une démarche sans cesse à renouveler, qui ne peut se limiter à une seule journée ni à une seule région du Québec. Les intervenants du réseau de la santé ainsi que la population générale doivent garder en tête que ces notions sont en constante évolution, ce qui implique une formation continue. L'AQPPEP planifie donc l'organisation d'autres journées de sensibilisation dans les prochaines années, dans différentes régions de la province.

À la suite de cette journée, des journalistes ont publié des articles dans L'Actualité médicale et dans Défi Schizophrénie sur la journée de sensibilisation et sur l'intervention précoce (Crépeau, 2006; Lepage, 2005)

\section{Site internet de l'AQPPEP}

Souhaiter rejoindre les personnes qui souffrent d'une psychose débutante requiert un médium qui leur est familier et facilement accessible. Internet est devenu un outil de travail et de loisir très puissant où l'on peut trouver de l'information sur quoi que ce soit d'un simple mouvement du doigt. Mais cette information si facile à obtenir est aussi inconsistante et comporte des risques: des données non scientifiques peuvent y être présentées de manière si professionnelle qu'elles semblent crédibles et prouvées. L'AQPPEP a créé son propre site pour contrer ce problème, compte tenu de la quasi absence de sites francophones scientifiques qui traitent des psychoses débutantes.

Le site Internet est accessible depuis novembre 2005, à l'adresse : www.aqppep.com.

La majeure partie du site est publique, et vise à aider les personnes aux prises avec des symptômes pré-psychotiques (c'est-à-dire prodromaux ou d' «état mental à risque de psychose») ou psychotiques, les familles et amis qui cherchent des informations sur le sujet, et les personnes qui interviennent auprès de personnes psychotiques et qui souhaitent être davantage documentés. On y retrouve de l'information sur l'Association, sur les psychoses, sur des lignes directrices de traitement ainsi qu'une bibliographie et des liens avec d'autres sites web appropriés. Le site présente aussi une description des différentes cliniques québécoises pour premiers épisodes psychotiques. Une deuxième section du site, réservée aux membres de l'Association, contient une bibliographie exhaustive et un agenda des formations et conférences traitant des psychoses.

Le site de l'Association sera mis à jour avec les développements récents dans ce domaine. Une version anglaise des textes devrait aussi 
être disponible sous peu, pour répondre aux besoins de la population anglophone du Québec et d'ailleurs, ainsi que pour faciliter les liens avec les autres associations similaires internationales.

\section{Communications}

Par le biais de présentation à divers congrès nationaux et internationaux (par exemple AMPQ, IEPA, etc.) et de publications, l'AQPPEP fait connaître ce qui se fait en termes d'intervention précoce au Québec et promeut son développement (L'Heureux et al., 2006; Abdel-Baki et al., 2004).

\section{Projets futurs}

L'AQPPEP continuera d'organiser pour la population générale, et les intervenants auprès des jeunes, des journées de sensibilisation aux divers enjeux reliés à la psychose débutante. De plus, des formations continues spécialisées sont régulièrement organisées pour affiner les connaissances des intervenants des différentes cliniques, et optimiser les traitements offerts aux jeunes patients atteints de psychose.

Un des principaux enjeux auxquels les cliniques pour premiers épisodes psychotiques font actuellement face est le manque de ressources professionnelles. Certaines cliniques débutent et tentent encore de rassembler un nombre critique d'intervenants pour offrir les services requis. D'autres cliniques existent déjà depuis plusieurs années et sont confrontées aux difficultés à référer les patients qui ont dépassé le stade d'intervention précoce, mais qui ont encore besoin d'un suivi psychiatrique. La continuité des soins serait idéale pour les patients, mais impossible à soutenir au long cours par une équipe dont la cohorte est sans cesse croissante. Enfin, la proportion grandissante de références à des programmes spécialisés par des omnipraticiens et des psychiatres, de même que la tendance réelle et souhaitée vers une détection plus précoce de la psychose ou même du risque imminent de psychose, mènent à une augmentation constante de références aux cliniques pour premiers épisodes psychotiques.

Le résultat est un combat constant entre qualité et quantité : le besoin de traitement intense interdisciplinaire, et le besoin d'intervention rapide pour toutes les personnes aux prises avec une première psychose imminente, aiguë ou récente. Ce combat, très exigeant et confrontant pour tous les professionnels, implique une meilleure organisation des services, et une allocation de ressources nettement supérieure à celle qui est actuellement consentie aux programmes pour premiers épisodes psychotiques. En regroupant les cliniques pour 
premiers épisodes, leurs membres ont la possibilité de partager leur expertise, d'optimiser l'organisation des services offerts et de préciser les standards de traitement souhaitables pour les patients. Par ailleurs, l'AQPPEP se donne principalement pour mandat de convaincre les hôpitaux et les instances gouvernementales de la nécessité cruciale d'une intervention spécialisée face aux psychoses débutantes, intervention qui implique plus que de simples rendez-vous avec un psychiatre et requiert davantage qu'une simple motivation. En effet, l'intervention auprès des personnes qui font face à une première psychose nécessite un nombre adéquat d'intervenants, bien formés dans leur champ d'expertise particulier, pouvant travailler dans des conditions qui permettent un investissement d'effort et de temps important et soutenu.

\section{NOTE}

1. Statuts et règlements de l'AQPPEP, 2004, 5.

\section{RÉFÉRENCES}

Abdel-BAKI, A., 2006, L'émergence de la psychose chez l'adolescent et le jeune adulte: réflexions sur l'intervention précoce et l'expérience australienne, Prisme, 45.

Abdel-Baki, A., Nicole, L., Roy, M-A., L'heureux, S., Gingras, N., 2004, Heureux événement: Naissance de l'AQPPEP!, Le Journal de l'AMPQ, 8,4 .

Addington, J., GLeEson, J., 2005, Implementing cognitive-behavioural therapy for first-episode psychosis, British Journal of Psychiatry, 187, suppl 48, 72-76.

AQPPEP, Statuts et règlements, juin 2004, 1-10.

BIRChwOOD, M., The critical period for early intervention, in Birchwood, M., Fowler, D., Jackson, H. J., eds., Early Intervention in Psychosis : A Guide to Concepts, Evidence, and Interventions, Wiley, New York, 28-63.

CRÉPEAU, C., 2006, Intervention précoce: Le Québec a du rattrapage à faire, Actualité médicale, 25 janvier, 16-17.

CRÉPEAU, C., 2006, Intervention précoce: Reconnaître et traiter un premier épisode psychotique, Actualité médicale, 25 janvier, 18-19.

Edwards, J., McGorry, P. D., 2002, Implementing Early Intervention in Psychosis - A Guide to Establishing Psychosis Services, Martin Dunitz ed, London. 
Ehmann, T., Mcewan, G. W., Honer, W. G., 2004, Best Care in Early Psychosis Intervention - Global Perspectives, Taylor and Francis, London.

FALLOON, I. R., 1992, Early intervention for first episodes of schizophrenia: a preliminary exploration, Psychiatry, 55, 4-15.

Harrigan, S. M., Mcgorry, P. D., Krstev, H., 2003, Does treatment delay in first-episode psychosis really matter?, Psychological Medicine, 33, 97110.

Larsen, T. K., Mcglashan, T. H., Johannessen, J. O., Fris, S., Guldberg, C., HaAhr, U., Horneland, M., Melle, I., Moe, L.C., Opjordsmoen, S., Simonsen, E., Vaglum, P., 2001, Shortened duration of untreated first episode of psychosis: changes in patient characteristics at treatment, American Journal of Psychiatry, 158, 1917-1919.

Lepage, J., 2005, Journée de sensibilisation à la psychose débutante: plus de 500 personnes présentes, Défi Schizophrénie, 10, 6, 3.

L'Heureux, S., Abdel-Baki, A., Nicole, L., Roy, M-A., Gingras, N., 2006, Increasing awareness of psychosis in Quebec: the "birth" of a provincial association of early psychosis clinics (AQPPEP), affiche AMPQ Québec et IEPA Birmingham (UK).

Linszen, D., Lenior, M., De HaAn, L., 1998, Early intervention, untreated psychosis and the course of early schizophrenia, British Journal of Psychiatry, Suppl, 172, 84-89.

Malla, A. M., Norman, R. M., 2001, Treating psychosis: is there more to early intervention than intervening early?, Canadian Journal of Psychiatry, 46, 645-648.

McGlashan, T. H., 1999, Duration of untreated psychosis in first-episode schizophrenia: marker or determinant of course ?, Biological Psychiatry, 46, 899-907.

McGorry, P. D., Chanen, A., Mccarthy, E., et al., 1991, Posttraumatic stress disorder following recent-onset psychosis, An unrecognized postpsychotic syndrome, Journal of Nervous and Mental Disease, 179, 253-258.

McGorry, P. D., 2000, The scope for preventive strategies in early psychosis: logic, evidence and momentum, in Birchwood M., Fowler D., Jackson H. J., eds., Early Intervention in Psychosis: A Guide to Concepts, Evidence, and Interventions, Wiley, New-York, 3-27. 


\section{ABSTRACT}

Improving detection and treatment of early psychosis in Québec: the Quebec association of early psychosis (l'Association québécoise des programmes pour premiers épisodes psychotiques, AQPPEP), sees to it

June 2004: the Quebec association of early psychosis programs, AQPPEP (Association québécoise des programmes pour premiers épisodes psychotiques), was created. The Association's objectives is to promote clinical and scientific discussions between health care professionals and researchers sharing an interest for people suffering from an early psychosis, and to improve earlier detection of psychosis. It also aims at increasing awareness of the problem in the general population and governments. To reach these goals, AQPPEP has organized the first early psychosis awareness day in Quebec and developed one of the rare French language web sites in this area. Finally, the Association is a tool to better face, in a concerted approach, some difficulties that many first episode clinics have to deal with, in order to share or develop common solutions.

\section{RESUMEN}

Mejorar la detección y el tratamiento de las psicosis iniciales en Quebec: La Asociación Quebequense de Programas Para los Primeros Episodios Psicóticos (AQPPEP) se encarga de ello

Junio de 2004: se crea la AQPPEP (Asociación Quebequense de Programas Para los Primeros Episodios Psicóticos) (http://www.aqppep. $\mathrm{com} /$ ). Su papel consiste en favorecer los intercambios clínicos y científicos entre los profesionales y los investigadores que se interesan en las personas que sufren de psicosis inicial, y permitir una detección más temprana de la psicosis. También busca la toma de conciencia de este problema por parte de la población y las instancias gubernamentales. Para ello, la AQPPEP organizó el primer día quebequense de sensibilización a la psicosis y desarrolló uno de los pocos sitios Internet francófonos en este campo. Con un espíritu de concertación, la Asociación constituye una herramienta para afrontar los problemas que encuentran varias clínicas para los primeros episodios psicóticos, además de compartir y desarrollar soluciones comunes entre ellas. 


\section{RESUMO}

Melhorar a detecção e o tratamento de psicoses inicial no Quebec: criação da Associação Quebequense dos Programas para os Primeiros Episódios Psicóticos (AQPPEP)

Junho de 2004: Criação da AQPPEP, Associação Quebequense dos Programas para os Primeiros Episódios Psicóticos (Association québécoise des programmes pour premiers épisodes psychotiques http://www.aqppep.com/). Seu papel é favorecer os intercâmbios clínicos e científicos entre os profissionais e os pesquisadores que se interessam pelas pessoas que sofrem de psicose inicial, e permitir uma detecção mais precoce da psicose. Ela visa, igualmente, uma tomada de consciência desta problemática pela população e pelas instâncias governamentais. Para este fim, a AQPPEP organizou a primeira jornada quebequense de sensibilização à psicose, e desenvolveu um dos raros sites francófonos na Internet nesta área. Com um espírito de comum acordo, a Associação constitui uma ferramenta para enfrentar os problemas que várias clínicas encontram com respeito aos primeiros episódios psicóticos, compartilhar e desenvolver soluções comuns entre elas. 